\title{
Coherent control of stimulated emission inside one dimensional photonic crystals: strong coupling regime
}

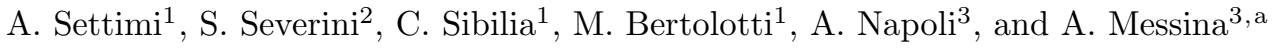 \\ 1 Dipartimento di Energetica, Università "La Sapienza" di Roma, via Scarpa 16, 00161 Roma, Italy \\ 2 Centro Interforze Studi Applicazioni Militari (CISAM) via della bigattiera, 56010 San Piero a Grado (PI), Italy \\ 3 Dipartimento di Scienze Fisiche ed Astronomiche, Università di Palermo, via Archirafi 36, 90123 Palermo, Italy
}

Received 15 December 2005

Published online 5 May 2006 - C EDP Sciences, Società Italiana di Fisica, Springer-Verlag 2006

\begin{abstract}
The present paper discusses the stimulated emission, in strong coupling regime, of an atom embedded inside a one dimensional (1D) Photonic Band Gap (PBG) cavity which is pumped by two counter-propagating laser beams. Quantum electrodynamics is applied to model the atom-field interaction, by considering the atom as a two level system, the e.m. field as a superposition of normal modes, the coupling in dipole approximation, and the equations of motion in Wigner-Weisskopf and rotating wave approximations. In addition, the Quasi Normal Mode (QNM) approach for an open cavity is adopted, interpreting the local density of states (LDOS) as the local density of probability to excite one QNM of the cavity; and therefore rendering this LDOS dependent on the phase difference of the two laser beams. In this paper we demonstrate that the strong coupling regime occurs at high values of the LDOS. In accordance with the results of the literature, the emission probability of the atom decays with an oscillatory behaviour, so that the atomic emission spectrum exhibits two peaks (Rabi splitting). The novelty of this work is that the phase difference of the two laser beams can produce a coherent control of both the oscillations for the atomic emission probability and, as a consequence, of the Rabi splitting in the emission spectrum. Possible criteria to design active delay lines are finally discussed.
\end{abstract}

PACS. $42.50 . \mathrm{Hz}$ Strong-field excitation of optical transitions in quantum systems; multiphoton processes; dynamic Stark shift - 32.80.Qk Coherent control of atomic interactions with photons - 42.70.Qs Photonic bandgap materials -42.79. Sz Optical communication systems, multiplexers, and demultiplexers

\section{Introduction}

It has been theoretically [1] and experimentally [2] demonstrated that emission processes, besides reflecting inherent properties of the atom, are sensitive to eventual boundary conditions and for example can be modified in a cavity whose size is comparable with the wavelength of the emitted light. This modification, including both enhancement or inhibition of emission processes, stems from the alteration of the field mode structure in the cavity with respect to that exhibited in free space. These effects are accounted for by considering the interaction between the atom and cavity modes [3].

When the density of states (DOS), interpretable as the density of probability to excite one eigenstate of the e.m. field, turns out to be a smooth function of frequency over the spectral range of the atomic transition, the rate of emission is described by Fermi's golden rule. On the other hand, abrupt changes in the DOS and photon localization effects [4] may drastically modify the emission dynamics. These modifications can be viewed as long time memory

\footnotetext{
a e-mail: messina@fisica.unipa.it
}

effects and manifestations of non-Markovian behaviour in the atom-reservoir interaction.

Strong modification in the DOS can be realized by means of photonic crystals which are dielectric materials characterized by refractive indices exhibiting strong periodic modulation leading to the appearance of not allowed [5] frequency bands (photonic band gap). Within a photonic band gap (PBG), the DOS is zero by definition. It has been suggested that the existence of such conditions might be accompanied by the inhibition of single-photon emission, classical light localization, a photon-atom bound state, fractionalized single-atom inversion, and anomalously large vacuum Rabi splitting [6].

The spontaneous decay of a two-level atom coupled to a narrow cavity resonance has been investigated rigorously in terms of the (Hermitian) modes of the "universe" rather than the (dissipative) quasi modes of the cavity by Lai et al. [7]. Special attention is paid to the strong-coupling regime (atomic line-width $\Gamma$ nearly equal to cavity resonance width $\gamma$ ), in which there are significant corrections to the golden rule. In particular, spontaneous emission decays rapidly for intermediate values of the quality factor $Q$ of the cavity resonance. 
In this paper, the emission processes in a $1 \mathrm{D}$ open cavity are analysed on the basis of the theory presented in reference [7]. In particular we discuss, in the strong coupling regime the stimulated emission of an atom located within a $1 \mathrm{D}-\mathrm{PBG}$ cavity pumped by two counter-propagating laser beams. As in reference [7], quantum electrodynamics is applied to model the atom-e.m. field coupling, by considering the atom as a two level system, the e.m. field a superposition of normal modes, the coupling in dipole approximation, and the equations of motion in WignerWeisskopf and rotating wave approximations. With respect to reference [7], the Quasi Normal Mode (QNM) approach for an open cavity is adopted: this means that the local density of states (LDOS) is interpreted as the local density of probability to excite one QNM of the cavity and as a consequence this local DOS becomes depending on the phase difference of the two laser beams.

\subsection{Quasi Normal Modes (QNMs)}

The problem of the field description inside a leaky cavity has been discussed by several authors. In particular Leung et al. [8] have introduced the description of the electromagnetic field in a one-side open and homogeneous cavity in terms of QNMs. Because of the leakage, the QNMs are characterized by complex eigenfrequencies and form an orthogonal basis only inside the cavity, according to a non-canonical metric.

In reference [9], the QNM treatment has been extended to double-side open and non-homogeneous cavities, in particular to 1D-PBG cavities.

A leaky cavity, viewed as a dissipative system, cannot be quantized [11] unless one considers the bath being part of the total universe in which energy is conserved [12]. Ho et al. [10] already made an essential first step towards the application of QNMs to cavity quantum electrodynamics phenomena.

In reference [13], the second quantization scheme based on the QNM theory has been extended to 1D-PBGs. In reference [14], the second QNM quantization is applied to $1 \mathrm{D}-\mathrm{PBG}$, excited by two counter-propagating pumps. The QNM commutation relations are not canonical and in addition they depends on the geometry of the open cavity and the phase-difference of the two pumps. In reference [15], the QNM theory is applied to discuss the stimulated emission, in weak coupling regime, for an atom, embedded inside a 1D-PBG, pumped by two counterpropagating laser beams. The main result of reference [15] is that the decay-time depends on the position of the dipole inside the cavity and can be controlled by the phase-difference of the two laser beams. Such a system is relevant for a single-atom, phase-sensitive, optical memory device on the atomic scale.

In this paper, the strong coupling regime is considered discussing the stimulated emission of an atom embedded inside a 1D-PBG cavity pumped by two counterpropagating laser beams. The main results of the paper consists in the demonstration that the strong coupling regime may be defined as the one associated to high values of the LDOS. We moreover find that, in accordance with the results of the literature, the emission probability of the atom decays with an oscillatory behaviour, so that the atomic emission spectrum is splitted into two peaks (Rabi splitting). The novelty of our paper with respect to previous results appeared in literature is that the phase difference of the two laser beams can be used to realize a coherent control of both the oscillations of the atomic emission probability and the Rabi splitting charactering the emission spectrum. Finally, some criteria are proposed to design the active cavity consisting of the $1 \mathrm{D}-\mathrm{PBG}$ plus the atom as an active delay line: suitable phase differences between the two laser beams allow to obtain a high transmission in a narrow pass band for a delayed pulse.

In Section 2, the coupling of an atom to an e.m. field is modelled via quantum electrodynamics equations, in analogy with the theory of an atom in the free space. In Section 3, the atom is embedded inside a leaky cavity; the LDOS is interpreted as the local density of probability to excite one QNM. In Section 4, the emission processes of the atom are modelled; in the stimulated emission, the LDOS depends on the phase difference of two counterpropagating laser beams. In Section 5 , the emission probability of the atom is discussed in strong coupling regime. In Section 6, the emission spectrum of the atom is characterized in terms of its poles. In Section 7 , we propose some criteria to design an active delay line. In Section 8 some conclusive remarks are reported and discussed.

\section{Coupling of an atom to an e.m. field}

Let us consider an atom, coupled to an e.m. field in the point $x_{0} \in U$, inside a one dimensional (1D) universe $U=$ $\{x \mid x \in[-L / 2, L / 2], L \rightarrow \infty\}$ of refractive index $n_{0}$, and an open cavity $C=\{x \mid x \in(0, d), d<L / 2\}$, with an inhomogeneous refractive index $n(x)$.

The atom is quantized as a two level system, oscillating at resonance $\Omega$ [11]. The e.m. field is quantized in terms of the $1 \mathrm{D}$ universe modes,

$$
\left\{\begin{array}{l}
g_{\lambda}(x)=\frac{1}{\sqrt{L}} \exp \left(i \omega_{\lambda} \sqrt{\rho_{0}} x\right) \\
\omega_{\lambda}=\lambda \frac{\pi}{(L / 2) \sqrt{\rho_{0}}} \lambda \in \mathbb{Z}
\end{array}\right.
$$

where $\rho_{0}=\left(n_{0} / c\right)^{2}$, being $c$ the speed of the light in vacuum. The atom is modelled by the dipole operator $\mu$ [11] along the polarization direction of the e.m. field, and the electric dipole approximation [12] is used to describe the coupling of the atom to the e.m. field.

At the initial time $(t=0)$, the atom is prepared in the excited state $|+\rangle$ and the e.m. field in the vacuum state $|\{0\}\rangle=\prod_{\lambda=-\infty}^{\infty}\left|0_{\lambda}\right\rangle$. With the above initial conditions, the system dynamics may be described using the basis states listed below, with their corresponding eigenvalues [7]:

$$
\begin{aligned}
& |+,\{0\}\rangle=|+\rangle|\{0\}\rangle, \quad \varepsilon_{+} \\
& \left|-, 1_{\lambda}\right\rangle=|-\rangle\left|1_{\lambda}\right\rangle, \quad \varepsilon_{-}+\hbar \omega_{\lambda}, \quad \lambda \in \mathbb{Z} .
\end{aligned}
$$


where $|+,\{0\}\rangle$ denotes the state for which the atom is in the upper state, and no photon exists in any e.m. mode; and $\left|-, 1_{\lambda}\right\rangle$ denotes the state for which the atom is in the lower state, one photon exists in the $\lambda$ th e.m. mode, while no photon exists in all the other e.m. modes.

\subsection{Quantum electrodynamics equations}

Assuming the initial condition $|+,\{0\}\rangle$, the state of the atom-field system at a time instant $t>0$, may be cast as follows:

$$
|\Psi(t)\rangle=c_{+}(t)|+,\{0\}\rangle+\sum_{\lambda=-\infty}^{\infty} c_{-, \lambda}(t)\left|-, 1_{\lambda}\right\rangle,
$$

where the probability amplitudes $c_{+}(t)$ and $c_{-, \lambda}(t)$ such that $c_{+}(0)=1$ and $c_{-, \lambda}(0)=0$ are introduced. The rotating wave approximation [12] is assumed. Starting from the time evolution equations for the probability amplitudes $c_{+}(t)$ and $c_{-, \lambda}(t)[7]$, that is

$$
\begin{aligned}
\frac{d c_{+}}{d t}= & \frac{\langle+|\mu|-\rangle}{\hbar} \sum_{\lambda=1}^{\infty} \sqrt{\frac{\hbar \omega_{\lambda}}{2 \varepsilon_{0} n_{0}^{2}}} g_{\lambda}\left(x_{0}\right) c_{-, \lambda}(t) \\
& \times \exp \left[-i\left(\omega_{\lambda}-\Omega\right) t\right] \\
\frac{d c_{-, \lambda}}{d t}= & \frac{\langle-|\mu|+\rangle}{\hbar} \sqrt{\frac{\hbar \omega_{\lambda}}{2 \varepsilon_{0} n_{0}^{2}}} g_{\lambda}^{*}\left(x_{0}\right) c_{+}(t) \exp \left[i\left(\omega_{\lambda}-\Omega\right) t\right],
\end{aligned}
$$

we may formally integrate the second one getting the following time evolution equation for the probability amplitude $c_{+}(t)$,

$$
\begin{aligned}
\frac{d c_{+}}{d t}=-\frac{M}{2 \varepsilon_{0} n_{0}^{2} \hbar^{2}} & \frac{1}{L} \sum_{\lambda=1}^{\infty} \hbar \omega_{\lambda} \\
& \times \int_{0}^{t} c_{+}(\tau) \exp \left[i\left(\omega_{\lambda}-\Omega\right)(\tau-t)\right] d \tau,
\end{aligned}
$$

where $\varepsilon_{0}$ is the dielectric constant in vacuum and $M=$ $|\langle+|\mu|-\rangle|^{2}$.

A correspondence can be established between the discrete modes of a 1D cavity with length $L$ and the continuous modes of a boundless universe with infinite length $L \rightarrow \infty$. The mode spectrum becomes continuous as $L \rightarrow \infty$, because of $\Delta \omega_{\lambda}=\pi /(L / 2) \sqrt{\rho_{0}} \approx d \omega \rightarrow 0$. In this limit, sums over discrete indices may be converted into integrals over a continuous frequency variable, i.e.

$$
\frac{1}{L} \sum_{\lambda=1}^{\infty} \Rightarrow \int_{0}^{\infty} d \omega \sigma^{(l o c)}\left(x_{0}, \omega\right)
$$

where $\sigma^{(l o c)}(x, \omega)$ is the local density of states (LDOS) interpretable as the density of probability that the e.m. field, in the point $x$, is excited on just one eigenstate, oscillating around the frequency $\omega[16]$. The range of integration over $\omega$ in the expression (2.6) strictly extends only from 0 to $\infty$, since the physical frequencies are defined to be positive. However, the range can be extended from $-\infty$ to $\infty$ without significant errors, since most optical experiments use a narrow band source $B$ [17], i.e. $B \ll \omega_{c}, \omega_{c}$ being the central frequency of the bandwidth $B$. Thus, the time evolution equation (2.5) becomes,

$$
\frac{d c_{+}}{d t}=\int_{0}^{t} d \tau \cdot K\left(x_{0}, t-\tau\right) c_{+}(\tau)
$$

$K(x, t)$ being the kernel function, defined as:

$K(x, t)=-\frac{M}{2 \varepsilon_{0} n_{0}^{2} \hbar^{2}} \int_{-\infty}^{\infty} d \omega \hbar \omega \sigma^{(l o c)}(x, \omega) \exp [-i(\omega-\Omega) t]$.

From (2.8) the kernel function depends very strongly on the LDOS through $\sigma^{(l o c)}(x, \omega)$ which can be reinterpreted as the photon density of states in the reservoir. In essence, equation (2.8) is a measure of the photon reservoir's memory of its previous state, on a time scale of evolution of the atomic system, so $K(x, t)$ can be interpreted as memory kernel of the reservoir.

\subsection{Atom in the free space}

If the atom is localized at a point $x_{0}$ which is outside the open cavity, i.e. $x_{0}<0$ or $x_{0}>d$, then the local DOS $\sigma^{(l o c)}(x, \omega)$ is referred to the free space $[12,15]$ :

$$
\sigma^{(l o c)}(x, \omega)=\sigma_{\text {free-space }}(\omega)=\sigma_{\text {ref }}=\frac{\sqrt{\rho_{0}}}{2 \pi} .
$$

The emission probability of the atom decays exponentially in the free space,

$$
\left|c_{+}(t)\right|^{2}=\exp \left(-\Gamma_{0} t\right), \quad t \geq 0,
$$

$\Gamma_{0}$ being the atomic decay rate:

$$
\Gamma_{0}=\frac{M}{\hbar} \frac{\sqrt{\rho_{0}}}{\varepsilon_{0} n_{0}^{2}} \Omega /\left[1+\frac{1}{4}\left(\frac{M}{\hbar} \frac{\sqrt{\rho_{0}}}{\varepsilon_{0} n_{0}^{2}}\right)^{2}\right] .
$$

Since the free space is an infinitely broad photon reservoir (flat spectrum), its response should be instantaneous and the memory effect, associated with emission dynamics, is infinitesimally short compared to all time intervals of interest. Such interactions are said to be Markovian [12]. In other words, in the free space, the population of the excited state eventually decays to the ground level, independent of the strength of a driving field. This is a general result valid for almost any broadband smoothly varying DOS.

In view of the analysis of the next section we now introduce the following parameter:

$$
R=\frac{\Gamma_{0}}{\Omega} \cong \frac{M}{\hbar} \frac{\sqrt{\rho_{0}}}{\varepsilon_{0} n_{0}^{2}}
$$

which may be interpreted as degree of atom-field coupling, the kernel function (2.8) being expressible as:

$K(x, t)=-\frac{R}{2 \sqrt{\rho_{0}}} \int_{-\infty}^{\infty} d \omega \cdot \omega \sigma^{(l o c)}(x, \omega) \exp [-i(\omega-\Omega) t]$. 


\section{Atom inside an open cavity}

Let us suppose $0<x_{0}<d$, corresponding to the atom embedded in a point $x_{0}$ of the open and inhomogeneous cavity with refractive index $n(x)$. If the atom, with resonance frequency $\Omega$, is coupled to the $n$th QNM, oscillating at frequency $\operatorname{Re}\left(\omega_{n}\right)$, then this coupling is characterized by the frequency detuning:

$$
\Delta_{n}=\frac{\operatorname{Re} \omega_{n}-\Omega}{R} .
$$

\subsection{Density of states (DOS) as density of probability to excite one QNM}

If two counter-propagating pumps are filtered at the atomic resonance $\Omega \approx \operatorname{Re} \omega_{n}$, only the $n$th QNM can be excited and not the other QNMs, because only the $n$th QNM oscillates at the frequency $\operatorname{Re} \omega_{n}$ within the narrow range $2\left|\operatorname{Im} \omega_{n}\right| \ll\left|\operatorname{Re} \omega_{n}\right|$, so distant enough from the other QNMs [9]. The local density of probability that, around the point $x$, the e.m. field is actually excited on the $n$th $\mathrm{QNM}$ is [15]

$$
\sigma_{n}^{(l o c)}(x, \omega)=\frac{1}{I_{n}} \sigma_{n}(\omega) \rho(x)\left|f_{n}^{N}(x)\right|^{2}, \quad \forall x \mid 0<x<d,
$$

directly related to the (integral) density of probability $\sigma_{n}(\omega)$ for the $n$th QNM. In equation $(3.2), \rho(x)=$ $[n(x) / c]^{2}, I_{n}$ denotes a suitable overlapping integral [13], and $f_{n}^{N}(x)=f_{n}(x) \sqrt{2 \omega_{n} /\left\langle f_{n} \mid f_{n}\right\rangle}$ is the normalized QNM function, $\left\langle f_{n} \mid f_{n}\right\rangle$ being the QNM norm.

In order to discuss the spontaneous emission, the two pumps are modelled as vacuum fluctuations, lying on the ground state of the e.m. field [12-14]. Inside the open cavity the (integral) density of probability that the $n$th QNM is excited can be expressed as [15]:

$$
\begin{aligned}
\sigma_{n}^{(I)}(\omega) & =\frac{1}{d} \int_{0}^{d} \sigma_{n}^{(I)}(x, \omega) d x \\
& =\alpha_{n} \frac{d}{2 \pi} \frac{I_{n}^{2}\left|\operatorname{Im} \omega_{n}\right|}{\left(\omega-\operatorname{Re} \omega_{n}\right)^{2}+\operatorname{Im}^{2} \omega_{n}} .
\end{aligned}
$$

The normalization constant $\alpha_{n}$ is deducible from the following condition:

$$
\int_{\operatorname{Re} \omega_{\mathrm{n}}-\left|\operatorname{Im} \omega_{\mathrm{n}}\right|}^{\operatorname{Re} \omega_{\mathrm{n}}+\left|\operatorname{Im} \omega_{\mathrm{n}}\right|} \sigma_{n}^{(I)}(\omega) d \omega+\int_{-\operatorname{Re} \omega_{\mathrm{n}}-\left|\operatorname{Im} \omega_{\mathrm{n}}\right|}^{-\operatorname{Re} \omega_{\mathrm{n}}+\left|\operatorname{Im} \omega_{\mathrm{n}}\right|} \sigma_{n}^{(I)}(\omega) d \omega=\frac{1}{d} .
$$

As from equation (3.3), the density of probability for the $n$th QNM, due to vacuum fluctuations, is a Lorentzian function, with real and imaginary parts of the $n$th QNM frequency as parameters. The overlapping integral $I_{n}$ is related to the statistical weight of the $n$th QNM in the DOS. In equation (3.4), the density of probability $\sigma_{n}^{(I)}(\omega)$ is integrated also in the range of negative frequencies $\omega \in\left[-\operatorname{Re} \omega_{n}-\left|\operatorname{Im} \omega_{n}\right|,-\operatorname{Re} \omega_{n}+\left|\operatorname{Im} \omega_{n}\right|\right]$, since the QNM frequency $\omega_{n}$, with $\operatorname{Re}\left(\omega_{n}\right)>0$, is represented also by frequency $\omega_{-n}=-\omega_{n}^{*}$, with $\operatorname{Re}\left(\omega_{-n}\right)<0[9]$.

In order to discuss the stimulated emission, the two pumps are modelled as two laser beams, lying on a coherent state [12-14]. When the refractive index $n(x)$ satisfies the symmetry property $n(d / 2-x)=n(d / 2+x)$, the density of probability that inside the cavity the e.m. field is excited on the $n$th QNM can be expressed as [15]:

$$
\sigma_{n}^{(I I)}(\omega)=\sigma_{n}^{(I)}(\omega)\left[1+(-1)^{n} \cos \Delta \varphi\right]
$$

Equation (3.5) shows that the density of probability for the $n$th QNM, due to two counter propagating laser beams, can be controlled by the phase-difference $\Delta \varphi$ of the two laser beams.

\section{Emission processes of the atom}

If the atom is at the point $x_{0}$ of the open and inhomogeneous cavity, i.e. $0<x_{0}<d$, and the coupling with electromagnetic field is confined only to the $n$th QNM $\left[\omega_{n}, f_{n}^{N}(x)\right]$ of the open cavity, then the local density of probability $\sigma_{n}^{(l o c)}(x, \omega)$, is linked to the integral density probability $\sigma_{n}(\omega)$ by equation (3.2) and the emission processes of the atom are characterized by a kernel function $K(x, t)$ which can be expanded as (see Eq. (2.13))

$$
\begin{aligned}
& K_{n}(x, t)=-\frac{R}{2 \sqrt{\rho_{0}}} \frac{1}{I_{n}} \rho(x)\left|f_{n}^{N}(x)\right|^{2} \\
& \times \int_{-\infty}^{\infty} d \omega \cdot \omega \sigma_{n}(\omega) \exp [-i(\omega-\Omega) t], \forall x \mid 0<x<d .
\end{aligned}
$$

\subsection{Spontaneous emission: DOS corresponding to vacuum fluctuations}

If the open cavity is in presence of only vacuum fluctuations, which are filtered at the atomic resonance $\Omega$, close to $n$th QNM frequency of the cavity $\left(\Omega \approx \operatorname{Re} \omega_{n}\right)$, then the integral density of probability $\sigma_{n}^{(I)}(\omega)$ for the $n$th QNM is expressed by equations $(3.3,3.4)$ and the spontaneous emission of the atom is characterized by a time evolution equation (see Eq. (2.7)) where the kernel function $K_{n}(x, t)$ can be specified as (see Eq. (4.1))

$$
\begin{aligned}
& K_{n}^{(I)}(x, t)=-\frac{R}{2 \sqrt{\rho_{0}}} \frac{1}{I_{n}} \rho(x)\left|f_{n}^{N}(x)\right|^{2} \alpha_{n} \frac{d}{2 \pi} I_{n}^{2} \exp (i \Omega t) \\
& \times i \sqrt{2 \pi}\left[\frac{1}{\sqrt{2 \pi}} \int_{-\infty}^{\infty}(-\omega) \frac{\left|\operatorname{Im} \omega_{n}\right|}{\left(\omega-\operatorname{Re} \omega_{n}\right)^{2}+\operatorname{Im}^{2} \omega_{n}} \exp (-i \omega t) d \omega\right] .
\end{aligned}
$$


It is easy to transform the following signal $x(t)$ in the Fourier domain [18],

$$
\begin{aligned}
x(t) & =\frac{1}{2} \frac{d}{d t}\left[e^{-\left(\left|\operatorname{Im} \omega_{n}\right|+i \operatorname{Re} \omega_{n}\right)|t|}\right] \\
& \Rightarrow X(\omega)=\frac{1}{\sqrt{2 \pi}} \int_{-\infty}^{\infty} x(t) \exp (i \omega t) d t \\
& =-\frac{i \omega}{\sqrt{2 \pi}} \frac{\left|\operatorname{Im} \omega_{n}\right|}{\operatorname{Im}^{2} \omega_{n}+\left(\omega-\operatorname{Re} \omega_{n}\right)^{2}} .
\end{aligned}
$$

The kernel function for the spontaneous emission process, using equations (4.3-4.2), results:

$$
\begin{aligned}
K_{n}^{(I)}(x, t)=-\frac{1}{4} \frac{R}{\sqrt{\rho_{0}}} & \alpha_{n}\left(d \cdot I_{n}\right) \omega_{n} \rho(x)\left|f_{n}^{N}(x)\right|^{2} \\
& \times \exp \left[-i\left(\omega_{n}-\Omega\right) t\right], \quad \forall t \geq 0 .
\end{aligned}
$$

Deriving under the integral sign [18] into equation (2.7), we get

$$
\frac{d^{2} c_{+}}{d t^{2}}=K_{n}^{(I)}\left(x_{0}, t=0\right) c_{+}(t)+\int_{0}^{t} d \tau \frac{\partial K_{n}^{(I)}}{\partial t} c_{+}(\tau),
$$

and then deriving once more equation (4.4), sampled in the $x_{0}$ point, with respect to time,

$$
\frac{\partial K_{n}^{(I)}}{\partial t}=-i\left(\omega_{n}-\Omega\right) K_{n}^{(I)}\left(x_{0}, t\right), \quad t \geq 0,
$$

we have, after some algebra, a second order differential equation in time for the probability of spontaneous emission:

$$
\begin{aligned}
& \frac{d^{2} c_{+}}{d t^{2}}+i\left(\omega_{n}-\Omega\right) \frac{d c_{+}}{d t}-K_{n}^{(I)}\left(x_{0}, t=0\right) c_{+}(t)=0 \\
& c_{+}(0)=1,\left.\frac{d c_{+}}{d t}\right|_{t=0} \propto c_{-, n}(0)=0 .
\end{aligned}
$$

\subsection{Stimulated emission: DOS depending on the phase difference of two counter-propagating laser-beams}

If the open cavity is coherently pumped by two counter propagating laser beams with a phase difference $\Delta \varphi$, which are tuned at the atomic resonance $\Omega$, closed to the $n$th QNM frequency $\left(\Omega \approx \operatorname{Re} \omega_{n}\right)$, then the density of probability $\sigma_{n}^{(I I)}(\omega)$ for the $n$th QNM, corresponding to the two laser beams, is linked to $\sigma_{n}^{(I)}(\omega)$, calculated in presence of vacuum fluctuations, by equation (3.5), and the stimulated emission of the atom is characterized by a kernel function $K_{n}(x, \omega)$, which can be specified as (see Eq. (4.2))

$$
K_{n}^{(I I)}(x, t)=k_{n}^{(I)}(x, t)\left[1+(-1)^{n} \cos \Delta \varphi\right] .
$$

In terms of the frequency detuning (3.1), the quantity $\left(\omega_{n}-\Omega\right)$ can be re-expressed as $\left(\omega_{n}-\Omega\right)=\left(\operatorname{Re} \omega_{n}-\right.$ $\Omega)+i \operatorname{Im} \omega_{n}=R \Delta_{n}+i \operatorname{Im} \omega_{n}$ and the second order differential equation for the probability of emission becomes

$$
\frac{d^{2} c_{+}}{d t^{2}}+i\left(R \Delta_{n}+i \operatorname{Im} \omega_{n}\right) \frac{d c_{+}}{d t}-K_{n}\left(x_{0}, t=0\right) c_{+}(t)=0 .
$$

\section{Emission probability of the atom}

The algebraic equation associated to the Cauchy problem (4.9), with initial conditions as in equation (4.7), i.e.

$$
p^{2}+\left(R \Delta_{n}+i \operatorname{Im} \omega_{n}\right) p+K_{n}\left(x_{0}, t=0\right)=0,
$$

is solved by two roots,

$$
p_{1,2}=\frac{\left(R \Delta_{n}+i \operatorname{Im} \omega_{n}\right)}{2}\left[-1 \pm \sqrt{1-\frac{4 K_{n}\left(x_{0}, t=0\right)}{\left.\left(R \Delta_{n}+i \operatorname{Im} \omega_{n}\right)^{2}\right)}}\right]
$$

which allow to express the particular integral of the differential equation (4.9):

$$
c_{+}(t)=\frac{p_{2}}{p_{2}-p_{1}} \exp \left(i p_{1} t\right)-\frac{p_{1}}{p_{2}-p_{1}} \exp \left(i p_{2} t\right) .
$$

The coupling between atom and the $n$th QNM is established in strong coupling regime when the particular integral (5.3) presents an oscillatory behaviour, and then the two roots (5.2), of the associated algebraic equation (5.1), are complex conjugated [7].

\subsection{Strong coupling regime}

Let us consider the spontaneous emission in order to discuss the atom - $n$th QNM coupling in strong regime. Since the following condition is valid,

$$
\begin{aligned}
4\left|\frac{K_{n}^{(I)}\left(x_{0}, t=0\right)}{\left(R \Delta_{n}+i \operatorname{Im} \omega_{n}\right)^{2}}\right| & <\frac{R}{d \cdot I_{n}} \frac{\sigma_{n}^{(I)}\left(x_{0}, \Omega\right)}{\sigma_{\text {free-space }}} \\
& \cong R \frac{\sigma_{n}^{(I)}\left(x_{0}, \Omega\right)}{\sigma_{\text {free-space }}}
\end{aligned}
$$

we can talk about strong coupling regime if [7]:

$$
4\left|\frac{K_{n}\left(x_{0}, t=0\right)}{\left(R \Delta_{n}+i \operatorname{Im} \omega_{n}\right)^{2}}\right|>1 \Rightarrow \frac{\sigma_{n}^{(I)}\left(x_{0}, \Omega\right)}{\sigma_{\text {free-space }}}>\frac{1}{R} .
$$

As from equation (5.5), the strong coupling regime is established when the density of probability (3.3) inside the open cavity and sampled at the atomic resonance, in units of the DOS (2.9) referred to the free space, exceeds the inverse of the atomic parameter $R$ (see Eq. (2.12)). This legitimates the interpretation of the parameter $R$ as a degree of atom field coupling:the more $R$ is large, in fact, the more equation (5.5) is satisfied. In the hypothesis of strong coupling regime (5.5), the two roots (5.2) become complex conjugated,

$$
p_{1,2} \cong-\frac{R \Delta_{n}+i \operatorname{Im} \omega_{n}}{2} \pm i \sqrt{K_{n}^{(I)}\left(x_{0}, t=0\right)},
$$


and the particular integral (5.3) presents an oscillatory behaviour:

$$
\begin{aligned}
& c_{+}(t) \cong \exp (\left.-i \frac{R \Delta_{n}+i \operatorname{Im} \omega_{n}}{2} t\right) \\
& \times\left\{\cosh \left[\sqrt{K_{n}^{(I)}\left(x_{0}, t=0\right) t}\right]\right. \\
&\left.+i \frac{R \Delta_{n}+i \operatorname{Im} \omega_{n}}{2 \sqrt{K_{n}^{(I)}\left(x_{0}, t=0\right)}} \sinh \left[\sqrt{K_{n}^{(I)}\left(x_{0}, t=0\right) t}\right]\right\} .
\end{aligned}
$$

In fact (see Eq. (4.4)) $K_{n}^{(I)}\left(x_{0}, t=0\right)=-K_{n}^{(I)}\left(x_{0}, t=\right.$ $0)$. The oscillatory behaviour may be interpreted as the emission and re-absorption of one photon; the net decay rate is then determined by the rate of photon leakage, i.e. $\left|\operatorname{Im} \omega_{n}\right| / 2$.

Now, let us consider the stimulated emission; again the coupling between atom and the $n$th QNM can be discussed in strong regime. If the two counter propagating laser beams have a phase difference $\Delta \varphi$, the atom $n$th QNM coupling is characterized by the kernel function (4.8). In the hypothesis of strong coupling, expressed by a condition similar to equation (5.5), the particular integral (5.3) presents an oscillatory behaviour,

$$
\begin{aligned}
c_{+}(t) \cong & \exp \left(-i \frac{R \Delta_{n}+i \operatorname{Im} \omega_{n}}{2} t\right)\left\{\cosh \left[\sqrt{K_{n}^{(I I)}\left(x_{0}, t=0\right) t}\right]\right. \\
& \left.+i \frac{R \Delta_{n}+i \operatorname{Im} \omega_{n}}{2 \sqrt{K_{n}^{(I I)}\left(x_{0}, t=0\right)}} \sinh \left[\sqrt{K_{n}^{(I I)}\left(x_{0}, t=0\right) t}\right]\right\},
\end{aligned}
$$

where $K_{n}^{(I I)}\left(x_{0}, t=0\right)$ is connected to the phase difference $\Delta \varphi$ by equation (4.8). The number of oscillations for the atomic emission probability depends on the position of the atom inside the cavity and can be controlled by the phasedifference of the two laser-beams. The following condition,

$$
1+(-1)^{n} \cos \Delta \varphi=0,
$$

is verified whether the atom is coupled to an odd QNM, i.e. $n=1,3, \ldots$ and the two laser beams are in phase, i.e. $\Delta \varphi=0$, or the atom is coupled to an even QNM, i.e. $n=$ $0,2, \ldots$ and the two laser beams are opposite in phase, i.e. $\Delta \varphi=\pi$. If equation (5.9) is satisfied, the emission probability is over-damped inside the whole cavity; even if in strong coupling, no oscillation occurs (see Eq. (5.8)):

$$
\begin{aligned}
& K_{n}^{(I I)}\left(x_{0}, t=0\right)=0 \Rightarrow\left|b_{+}(t)\right|^{2}= \\
& \quad \exp \left(\frac{\left|\operatorname{Im} \omega_{n}\right|^{2}}{4} t\right)\left[\left(1+\frac{\left|\operatorname{Im} \omega_{n}\right|}{2} t\right)^{2}+\left(\frac{R \Delta_{n}}{2} t\right)^{2}\right] .
\end{aligned}
$$

\section{Emission spectrum of the atom}

At the initial time $(t=0)$, the atom, located in the point $x_{0}$, is in its upper state and no photon exists in any normal mode, i.e. $c_{+}\left(x_{0}, t=0\right)=1$; when the atomic decay has occurred $(t=\infty)$, the coefficient of probability $c_{-, \lambda}\left(x_{0}, t\right)$ to find the atom in its lower state and one photon in the $\lambda$ th e.m. mode and no photon in all the other modes can be derived from equation (2.4):

$$
\begin{aligned}
c_{-, \lambda}\left(x_{0}, t=\infty\right) & =-\frac{\langle-|\mu|+\rangle}{\hbar} \sqrt{\frac{\hbar \omega_{\lambda}}{2 \varepsilon_{0} n_{0}^{2}}} g_{\lambda}^{*}\left(x_{0}\right) \\
& \times \int_{0}^{\infty} c_{+}\left(x_{0}, t\right) \exp \left[i\left(\omega_{\lambda}-\Omega\right) t\right] d t .
\end{aligned}
$$

In terms of the Laplace transform for the probability coefficient $c_{+}\left(x_{0}, t\right)$,

$$
C_{+}\left(x_{0}, s\right)=\frac{1}{\sqrt{2 \pi}} \int_{0}^{\infty} c_{+}\left(x_{0}, t\right) \exp (-s t) d t,
$$

equation (6.1) is also re-expressed as:

$$
\begin{aligned}
c_{-, \lambda}\left(x_{0}, t=\infty\right) & =-\frac{\langle-|\mu|+\rangle}{\hbar} \sqrt{\frac{\hbar \omega_{\lambda}}{2 \varepsilon_{0} n_{0}^{2}}} \\
& \times g_{\lambda}^{*}\left(x_{0}\right) \sqrt{2 \pi} C_{+}\left[x_{0}, s=i\left(\omega_{\lambda}-\Omega\right)\right] .
\end{aligned}
$$

If the decay has occurred $(t=\infty)$, the atomic emission spectrum can be defined as the density of probability that the atom in the point $x_{0}$ has emitted at the frequency $\omega[7]$,

$$
W\left(x_{0}, \omega\right)=\sum_{\lambda=1}^{\infty}\left|c_{-, \lambda}\left(x_{0}, t=\infty\right)\right|^{2} \delta\left(\omega-\omega_{\lambda}\right),
$$

$\delta(t)$ being the delta distribution of Dirac. Inserting equation (6.3) into equation (6.4), yields

$$
\begin{aligned}
W\left(x_{0}, \omega\right)=\frac{M}{2 \varepsilon_{0} n_{0}^{2} \hbar^{2}} \frac{1}{L} \sum_{\lambda=1}^{\infty} \hbar \omega_{\lambda} 2 \pi & \\
& \times\left|C_{+}\left[x_{0}, i\left(\omega_{\lambda}-\Omega\right)\right]\right|^{2} \delta\left(\omega-\omega_{\lambda}\right) .
\end{aligned}
$$

If, according to equation (2.6), sums over discrete quantities are converted to integrals over continuous frequencies, using Dirac's delta properties, emission spectrum (6.5) can be reduced as

$W\left(x_{0}, \omega\right)=\alpha^{\prime} \cdot \frac{R}{2 \sqrt{\rho_{0}}} \omega \sigma^{(l o c)}\left(x_{0}, \omega\right) 2 \pi\left|C_{+}\left[x_{0}, i(\omega-\Omega)\right]\right|^{2}$,

$\alpha^{\prime}$ being a suitable constant of normalization and $\sigma^{(l o c)}(x, \omega)$ the local density of states (DOS). The atomic parameter $R$ is defined in equation (2.12).

The frequency range can be extended from $-\infty$ to $\infty$ without significant errors, since most optical experiments use a narrow band source [17]; the normalization constant $\alpha^{\prime}$ can be obtained applying the closure relation

$$
\int_{-\infty}^{\infty} W\left(x_{0}, \omega\right) d \omega=1
$$


directly coming from the probabilistic interpretation of emission spectrum (6.6). Let us suppose $0<x_{0}<d$ and $n\left(x_{0}\right)>n_{0}$, so that the atom is embedded inside the open cavity with inhomogeneous refractive in$\operatorname{dex} \rho(x)=[n(x) / c]^{2}$. The atom, having resonance frequency $\Omega$, is assumed to be coupled with the $n$th QNM, oscillating at the frequency $\operatorname{Re} \omega_{n}$; the coupling between atom and the $n$th QNM is characterized by the frequency detuning $\Delta_{n}$ (3.1). The normalization condition (6.7) reduces to:

$$
2 \int_{\Omega-\left|\operatorname{Im} \omega_{n}\right|}^{\Omega+\left|\operatorname{Im} \omega_{n}\right|} W_{n}\left(x_{0}, \omega\right) d \omega=1
$$

In equation (6.8), the integral over the positive frequencies is multiplied by a factor 2 for including the contribution of the negative frequencies (see comments after Eq. (3.4)).

As from equation (3.2), the local density of probability $\sigma_{n}^{(l o c)}(x, \omega)$ for the $n$th QNM is proportional to $\sigma_{n}(\omega)$. Inserting equation (3.2) in equation (6.6), the atomic emission spectrum becomes:

$$
\begin{aligned}
W_{n}\left(x_{0}, \omega\right)=\alpha_{n}^{\prime} \cdot & \frac{R}{2 \sqrt{\rho_{0}}} \frac{1}{I_{n}} \rho\left(x_{0}\right)\left|f_{n}^{(N)}\left(x_{0}\right)\right|^{2} \\
& \times \omega \sigma_{n}(\omega) 2 \pi \mid C_{+}^{(n)}\left[x_{0},\left.i(\omega-\Omega]\right|^{2}\right.
\end{aligned}
$$

$\alpha_{n}^{\prime}$ being normalization constant which satisfies equation (6.8).

The emission processes of the atom are characterized by a kernel function $K_{n}(x, t)$ which, in this case, can be written as reported in equation (4.4) and, for stimulated emission as in equation (4.8). Inserting equation (4.4) in equation (6.9), the emission spectrum (6.9) takes the expression:

$$
\begin{aligned}
\frac{W_{n}\left(x_{0}, \omega\right)}{\alpha_{n}^{\prime}}=- & \frac{2}{d \cdot I_{n}^{2}} \frac{K_{n}^{(I)}\left(x_{0}, t=0\right)}{\omega_{n}} \\
& \times \omega \frac{\sigma_{n}(\omega)}{K_{n}} 2 \pi\left|C_{+}^{(n)}\left[x_{0}, i(\omega-\Omega)\right]\right|^{2} .
\end{aligned}
$$

As from equation (6.10), the emission spectrum $W_{n}\left(x_{0}, \omega\right)$ depends on the density of probability $\sigma_{n}(\omega)$, as well as on the initial value of the kernel function $K_{n}^{(I)}\left(x_{0}, t=0\right)$.

By using Laplace transformation of the Cauchy problem (4.9), with initial conditions given by equation (4.7), we finally have [18]:

$$
\begin{aligned}
& C_{+}^{(n)}\left(x_{0}, i \xi\right)= \\
& \quad-\frac{i}{\sqrt{2 \pi}} \frac{\xi+R \Delta_{n}+i \operatorname{Im} \omega_{n}}{\xi^{2}+\left(R \Delta_{n}+i \operatorname{Im} \omega_{n}\right) \xi+K_{n}\left(x_{0}, t=0\right)},
\end{aligned}
$$

where $\xi$ is the shifted frequency $(\omega-\Omega), \Omega$ denoting the atomic resonance.

\subsection{Poles of the emission spectrum}

The emission spectrum of the atom can be described in terms of the poles, $p_{1}$ and $p_{2}$, which solve equation (5.1) and are expressed in equation (5.2). Under the hypothesis of strong coupling regime (see Eq. (5.5)), the atomic emission spectrum $W_{n}\left(x_{0}, \xi\right)$, that is a function of the shifted frequency $\xi=(\omega-\Omega)$, is characterized by two peaks, approximately centred in the resonances Re $p_{1}$ and Re $p_{2}$ and with bandwidths linked to $2\left|\operatorname{Im} p_{1}\right|$ and $2\left|\operatorname{Im} p_{2}\right|$; so, a Rabi splitting occurs, the two peaks being separated by:

$$
\Delta \xi=\operatorname{Re} p_{1}-\operatorname{Re} p_{2}
$$

Let us consider stimulated emission processes. The two counter propagating laser beams have a phase difference $\Delta \varphi$, so the emission spectrum $W_{n}\left(x_{0}, \xi\right)$ is described by a kernel function $K_{n}\left(x_{0}, t=0\right)$ linked to $\Delta \varphi$ (see Eq. (4.8)). As result, the Rabi splitting, besides depending on the position of the atom inside the cavity, can be controlled by the phase-difference of the two laser-beams.

If the operative condition defined by equation (5.9) is almost verified, i.e. $K_{n}\left(x_{0}, t=0\right) \approx 0$, the spectrum $W_{n}\left(x_{0}, \omega\right)$, function of the pure frequency $\omega$, is reduced to two almost superimposed pulses: a Lorentzian function centred in the $n$th QNM frequency $\operatorname{Re} \omega_{n}$, with a bandwidth $2\left|\operatorname{Im} \omega_{n}\right|$, superimposed to a Dirac distribution in the atomic resonance $\Omega \approx \operatorname{Re} \omega_{n}$, i.e. (see Eqs. (3.3-3.5))

$$
W_{n}\left(x_{0}, \omega\right) \approx \sigma_{n}^{(I I)}(\omega)+\frac{\alpha_{n}^{\prime \prime}}{d} \delta(\omega-\Omega) \rightarrow \delta(\omega-\Omega),
$$

where $\alpha_{n}^{\prime \prime}$ is normalization constant satisfying condition (6.8). In fact, the two poles, $\omega_{1}$ and $\omega_{2}$, are so simplified (see Eqs. (3.1) and (5.1, 5.2)):

$$
\begin{aligned}
& \omega_{1} \approx \operatorname{Re} \omega_{n}+i \operatorname{Im} \omega_{n} \\
& \omega_{2} \approx \Omega .
\end{aligned}
$$

\section{Criteria to design an active delay line}

Let us specify a photonic crystal (PC) as a symmetric Quarter-Wave (QW) 1D-PBG cavity to which the QNM theory has been applied in reference [9] and subsequent papers. Let us consider a symmetric QW 1D-PBG cavity with parameters $\lambda_{r e f}=1 \mu \mathrm{m}, N=5, n_{h}=2, n_{l}=1.5$ (see Fig. 1). This cavity is chosen because, it provides a simple physical situation within which to discuss some criteria for a design of an active delay line. Let us locate an atom in the centre of the $1 \mathrm{D}-\mathrm{PBG}$, i.e. $x_{0}=d / 2$ (see Fig. 1). As discussed in reference [13], for a symmetric QW 1D-PBG cavity with $\omega_{\text {ref }}$ as reference wavelength and $N$ periods, the $\left[0,2 \omega_{\text {ref }}\right)$ range includes $2 N+1$ QNMs which, excluding $\omega=2 \omega_{\text {ref }}$ are identified as $|n\rangle$, $n \in[0,2 N]$. If the atom is located in the centre $x_{0}$ of the $1 \mathrm{D}-\mathrm{PBG}$ cavity, it can be coupled to just one of the QNMs with an even $n$ : since in this position the QNM intensity $\left|f_{n}\right|^{2}$ has a maximum for even values of $n$ and is almost null for odd value of $n$.

The active cavity, consisting of the 1D-PBG cavity and one atom inside, is characterized by a global transmission spectrum $G\left(x_{0}, \omega\right)$ which is the product between the transmission spectrum of the 1D-PBG $|t(\omega)|^{2}$ and the emission 


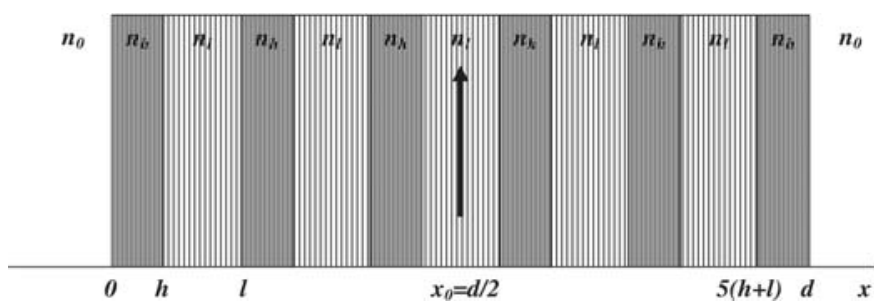

Fig. 1. Symmetric Quarter-Wave (QW) one dimensional (1D) Photonic Band Gap (PBG) cavity with $\lambda_{\text {ref }}=1 \mu \mathrm{m}$ as reference wavelength, $N=5$ periods, consisting of two layers with refractive indices $n_{h}=2$ and $n_{l}=1.5$ and lengths $h=\lambda_{\text {ref }} / 4 n_{h}$ and $l=\lambda_{\text {ref }} / 4 n_{l}$. Terminal layers of the symmetric QW 1D-PBG cavity with parameters: $n_{h}$ and $h=\lambda_{\text {ref }} / 4 n_{h}$. Length of the 1D-PBG cavity: $d=N(h+l)+h$. One atom is embedded in the centre of the $1 \mathrm{D}-\mathrm{PBG}$, i.e. $x_{0}=d / 2$.

spectrum $W\left(x_{0}, \omega\right)$ of the atom [in units of s], i.e.

$$
G\left(x_{0}, \omega\right)=W\left(x_{0}, \omega\right)|t(\omega)|^{2} \quad[\text { in units of } \mathrm{s}] .
$$

A "density of coupling" (DOC) for the active cavity $\sigma_{C}\left(x_{0}, \omega\right)$ can be defined as the density of probability that the atom, embedded in the point $x_{0}$, is coupled to just one QNM, oscillating around the frequency $\omega$; the DOC $\sigma_{C}\left(x_{0}, \omega\right)$ [in units of $\mathrm{s}^{2} / \mathrm{m}$ ] is the product between the DOS $\sigma(\omega)$ [in units of $\mathrm{s} / \mathrm{m}$ ] and the atomic emission spectrum $W\left(x_{0}, \omega\right)$. Moreover, an "acceleration of coupling" $a_{C}\left(x_{0}, \omega\right)$ inside the active cavity can be introduced as:

$$
\begin{aligned}
a_{C}\left(x_{0}, \omega\right)=\frac{1}{\sigma_{c}\left(x_{0}, \omega\right)} & =\frac{1}{W\left(x_{0}, \omega\right) \sigma(\omega)} \\
& \left.=\frac{v(\omega)}{W\left(x_{0}, \omega\right)} \quad \text { in units of } \mathrm{m} / \mathrm{s}^{2}\right]
\end{aligned}
$$

where $v(\omega)=1 / \sigma(\omega)$. In order to design the active cavity as an ideal delay line, the input pulse must be retarded with an high amplification but without any distortion: in a narrow pass band, the global transmission (7.1) must assume an high value and the acceleration of coupling (7.2) a quasi constant value.

As reported above, the atom embedded in the centre of the symmetric QW 1D-PBG cavity with $N=5$ periods (Fig. 1) can be coupled only to one QNM, oscillating next to an even transmission peak $n=0,2, \ldots, 2 N$. Let us suppose that the atom is coupled to the $(N+1)$ th QNM, next to the high frequency band edge, so the quality factor of the $1 \mathrm{D}-\mathrm{PBG}$ cavity is

$$
Q_{N+1}=\frac{\Omega}{\left|\operatorname{Im} \omega_{N+1}\right|}
$$

being $\Omega$ the resonance frequency of the atom. Let us assume to be in strong coupling regime; the directories for the active delay line can be satisfied by a suitable value of the coupling degree,

$$
R=\frac{\Gamma_{0}}{\Omega}
$$

$\Gamma_{0}$ denoting the decay rate of the atom in vacuum, and by a suitable value of the frequency detuning for the atom $(N+1)$ th QNM coupling,

$$
\Delta_{N+1}=\frac{\operatorname{Re} \omega_{N+1}-\Omega}{R} .
$$

In case of spontaneous emission, if a perfect tuning is assumed, i.e. $\Delta_{N+1}=0$, the atom oscillates at the frequency of the $(N+1)$ th QNM, i.e. $\Omega=\operatorname{Re} \omega_{N+1}$. Thus there exists (see Figs. $2 \mathrm{a}$ and $2 \mathrm{~b}$ ) a suitable value of the coupling degree $R$, i.e. $R^{*}=0.002506$, such that the two poles (Eqs. (5.2) and (4.4)) of the emission spectrum of the atom are distinct for $R>R^{*}$ or coincident for $0<R<R^{*}$. In other words, for $R>R^{*}$, a Rabi splitting (see Fig. 3a) occurs in the atomic emission spectrum (Eqs. (6.10, 6.11) and (4.4)), and so an oscillatory behaviour (see Fig. 4a) is present in the atomic emission probability (Eqs. (5.7) and (4.4)); however, for $0<R<R^{*}$, the emission spectrum consists of two superimposed peaks, and so the emission probability is over-damped. In order to find a Rabi splitting in strong coupling regime, consistent with experiments $\left(\Gamma_{0} \sim\left|\operatorname{Im} \omega_{N+1}\right|\right)$ [19], let us employ the degree of coupling:

$$
R=R_{N+1}=\frac{1}{Q_{N+1}} .
$$

The two poles of the spontaneous emission spectrum, shifted of the atomic resonance $\Omega$, are $\xi_{1}=0.06383+$ $i 0.01770$ and $\xi_{2}=-0.06383+i 0.01995$ in units of $\omega_{\text {ref }}$ (see Figs. 2a and 2b). They describe, in resonance and bandwidth, the two peaks of the emission spectrum, whose maxima are $W_{1}=21.87$ and $W_{2}=15.66$ in units of $\omega_{\text {ref }}$ (see Fig. 3a). Assuming that emission probability vanishes after the second oscillation, the decay time is $\tau=94.3$ in units of $1 / \omega_{\text {ref }}$ (see Fig. 4a). In this way, the active cavity has been designed just as a not ideal optical amplifier: an input pulse is amplified but distorted. In the spontaneous emission case, in fact, as plotted in Figures $5 \mathrm{a}$ and $5 \mathrm{~b}$ there exists a narrow pass band, i.e. $\xi=\omega-\Omega \approx(-0.06,0.06)$ (in units of $\omega_{\text {ref }}$ ), where the global transmission spectrum assumes relative high values, i.e. $G_{C, N+1} \in\left(G_{\min }, G_{\max }=(2.881,14,43)\right.$ in units of $1 / \omega_{\text {ref }}$, but the acceleration of coupling is subject to a modulation around the value $v_{C, N+1}=0.03445$ in units of $\omega_{\text {ref }} / v_{\text {ref }}$.

Let us consider now the case of stimulated emission; the atom inside the symmetric QW 1D-PBG cavity is excited by two counter propagating laser beams, so that the active delay line can be realized adding, as new degree of freedom, the phase difference $\Delta \varphi$ of the two laser beams. In case of stimulated emission, if a perfect tuning is assumed, i.e. $\Delta_{N+1}=0$, the atom oscillates still at the frequency of the $(N+1)$ th QNM, i.e. $\Omega=\operatorname{Re} \omega_{N+1}$. There exists (see Figs. 2c and $2 \mathrm{~d}$ ) a suitable range of the phase difference $\Delta \varphi$, i.e. $\left(\Delta \varphi_{1}, \Delta \varphi_{2}\right)=(2.747,3.524)$ in units of rads, such that the two poles (Eqs. (5.2) and (4.8)) of the emission spectrum of the atom are distinct for $\Delta \varphi<\Delta \varphi_{1}$ and $\Delta \varphi>\Delta \varphi_{2}$ but coincident for $\Delta \varphi_{1}<\Delta \varphi<\Delta \varphi_{2}$. In other words, for $\Delta \varphi<\Delta \varphi_{1}$ and $\Delta \varphi>\Delta \varphi_{2}$, a Rabi splitting (see Fig. 3a) occurs in the atomic emission spectrum 


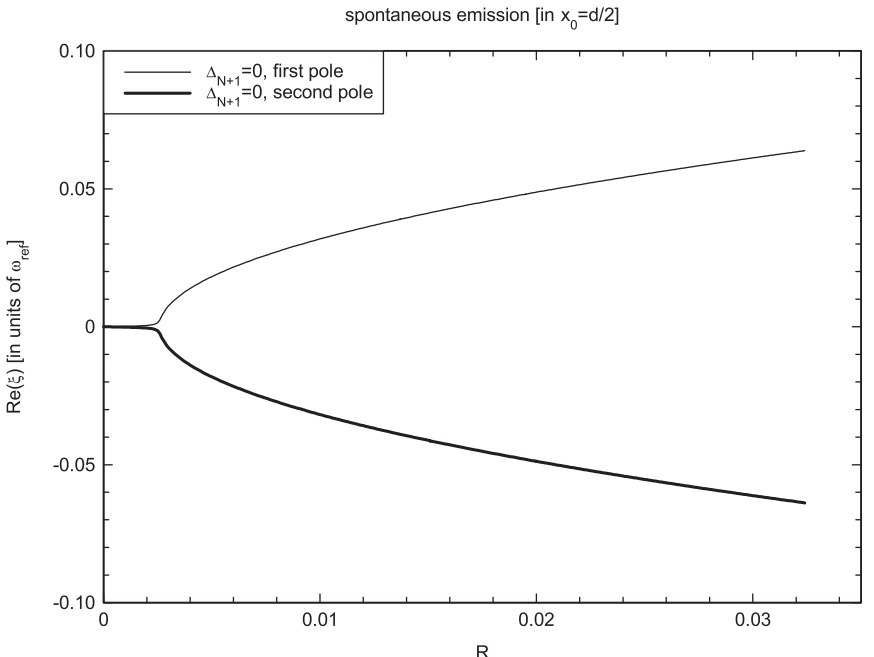

(a)

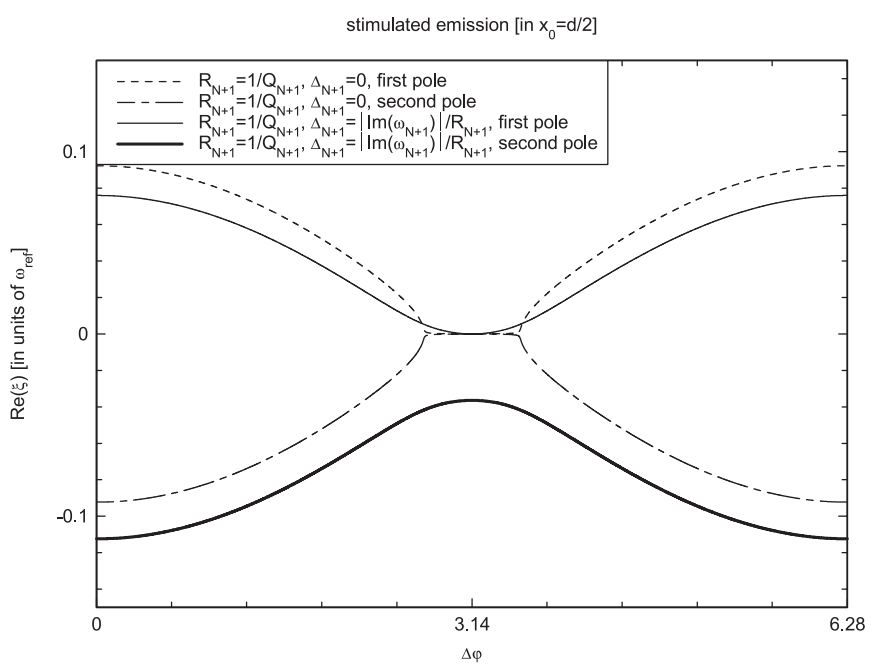

(c)

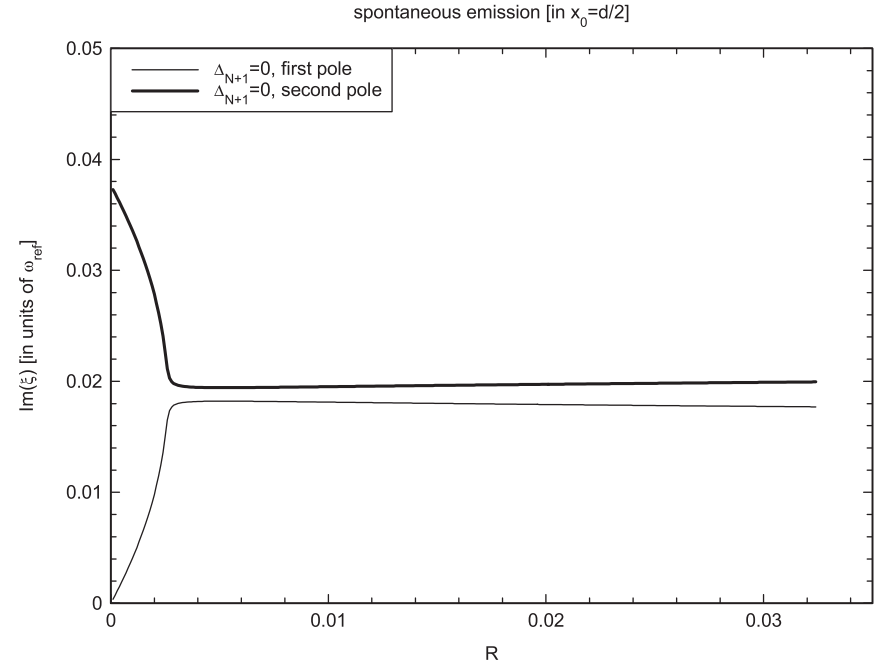

(b)

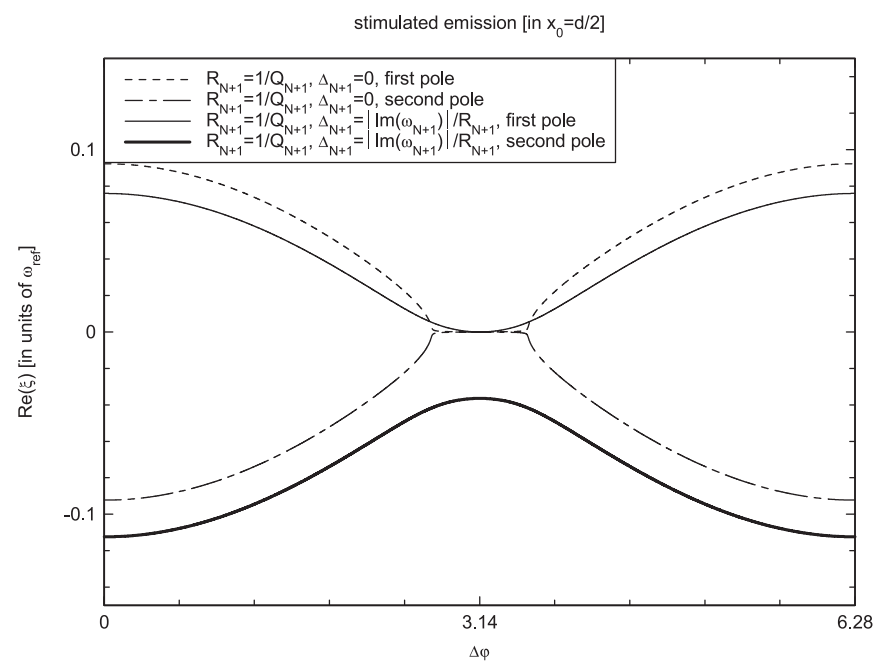

(d)

Fig. 2. If the atom embedded inside the $1 \mathrm{D}-\mathrm{PBG}$ cavity of Figure 1 oscillates at the $(\mathrm{N}+1)$ th Quasi Normal Mode (QNM), next to the high-frequency band edge [i.e. in perfect tuning $\Delta_{N+1},=0$, see Eq. (7.5)], the spontaneous emission in strong coupling regime can be characterized by the two poles of the emission spectrum of the atom, which poles are shifted of the atomic resonance $\Omega$ [see Eqs. (5.2) and (4.4)]; the real (Fig. 2a) and imaginary (Fig. 2b) parts, in units of the 1D-PBG reference frequency $\omega_{\text {ref }}$, are plotted as functions of the coupling degree $R=\Gamma_{0} / \Omega$, which is the ratio between the atomic decay-rate in vacuum $\Gamma_{0}$ and the resonance $\Omega$ [see Eq. (7.4)]. If two counter-propagating laser beams are tuned at the resonance $\Omega$ and the atom is coupled to the $(N+1)$ th QNM, (i.e. $Q_{N+1}=\Omega / / \operatorname{Im}\left[\omega_{N+1}\right] /$, see Eq. $(7.3)$ ), the stimulated emission in strong coupling (for $R_{N+1}=1 / Q_{N+1}$, see Eq. (7.6)) can be characterized by the two poles of the atomic emission spectrum, which poles are shifted of the resonance $\Omega$ [see Eqs. (5.2) and (4.8)]; whether the atom oscillating at the $(N+1)$ th QNM frequency (i.e. in perfect tuning $\Delta_{N+1}=0$ ) or at a frequency in the band gap next to the high frequency band edge [i.e. in detuning case $\Delta_{N+1}=\left|\operatorname{Im}\left[\omega_{N+1}\right]\right| / R_{N+1}$, see Eq. (7.9)], the real (Fig. 2c) and imaginary (Fig. 2d) parts, in units of the 1D-PBG reference frequency $\omega_{\text {ref }}$, are plotted as functions of the phase difference $\Delta \varphi$ between the two laser beams.

(Eqs. $(6.10,6.11)$ and $(4.8))$, and an oscillatory behaviour (see Fig. 4a) is present in the atomic emission probability (Eqs. (5.8) and (4.8)). For $\Delta \varphi_{1}<\Delta \varphi<\Delta \varphi_{2}$, the emission spectrum consists of two superimposed peaks and the emission probability is over-damped. As a consequence, the Rabi splitting and the oscillations of the decay time can be controlled by the phase difference of the two laser beams.
In order to obtain an ideal delay line by stimulated emission, the two laser beams must exceed in quadrature, i.e. $\Delta \varphi>\pi / 2$; with respect to spontaneous emission, the emission spectrum is characterized by a narrower Rabi splitting and the emission probability by a longer decay time. So, the active cavity, consisting of the 1D-PBG plus the atom, acts as an delay line, since the delay time of the active cavity is linked with the decay time of the atom 


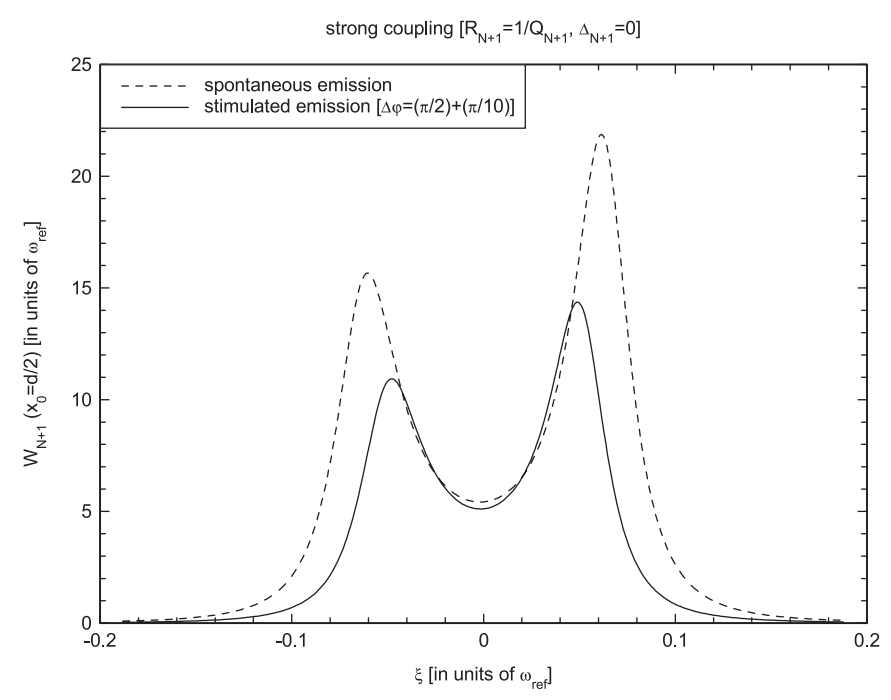

(a)

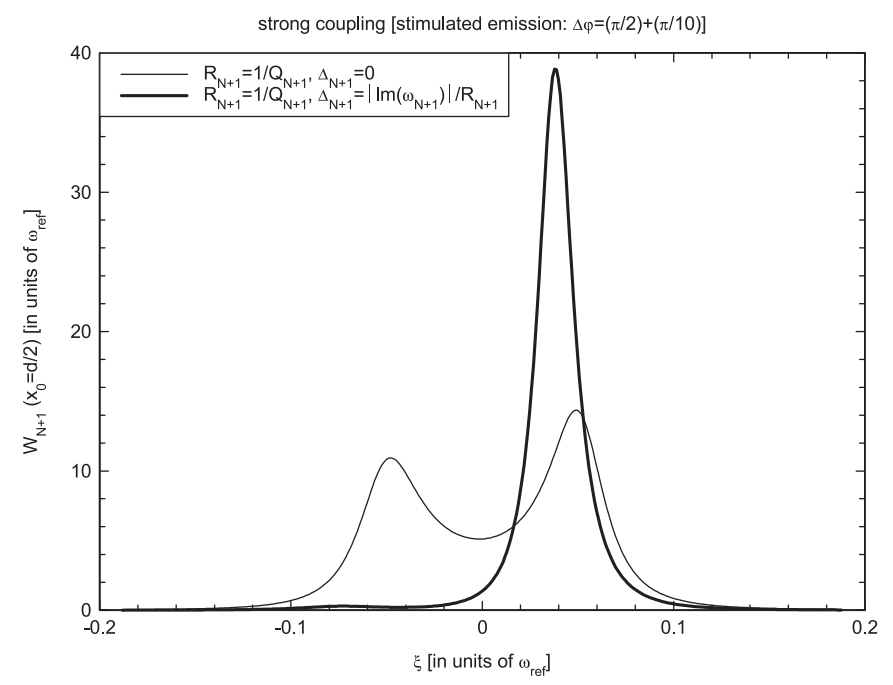

(b)

Fig. 3. Referring to the atom embedded inside the 1D-PBG cavity of Figure 1, the emission spectrum of the atom, in units of the $1 \mathrm{D}-\mathrm{PBG}$ reference frequency $\omega_{\text {ref }}$, is plotted as a function of the dimensionless shifted frequency $\xi=(\omega-\Omega) / \omega_{\text {ref }}$, being $\Omega$ the atomic resonance. The atom is coupled to the $(\mathrm{N}+1)$ th QNM frequency and the emission processes occur in strong coupling regime [for $R_{N+1}=1 / Q_{N+1}$ ]. In tuning hypothesis, as from Figure 3a, the atomic emission spectrum for spontaneous processes (see Eqs. $(6.10,6.11)$ ) is compared with the stimulated emission spectrum (see Eqs. $(6.10,6.11)$ ) when the $1 \mathrm{D}-\mathrm{PBG}$ is pumped by two laser beams with a suitable phase difference: $\Delta \varphi=(\pi / 2)+(\pi / 10)$ (see Eq. (7.7)). In the case of stimulated processes, as from Figure $3 \mathrm{~b}$, the atomic emission spectrum in perfect tuning is compared with the emission spectrum in detuning case.

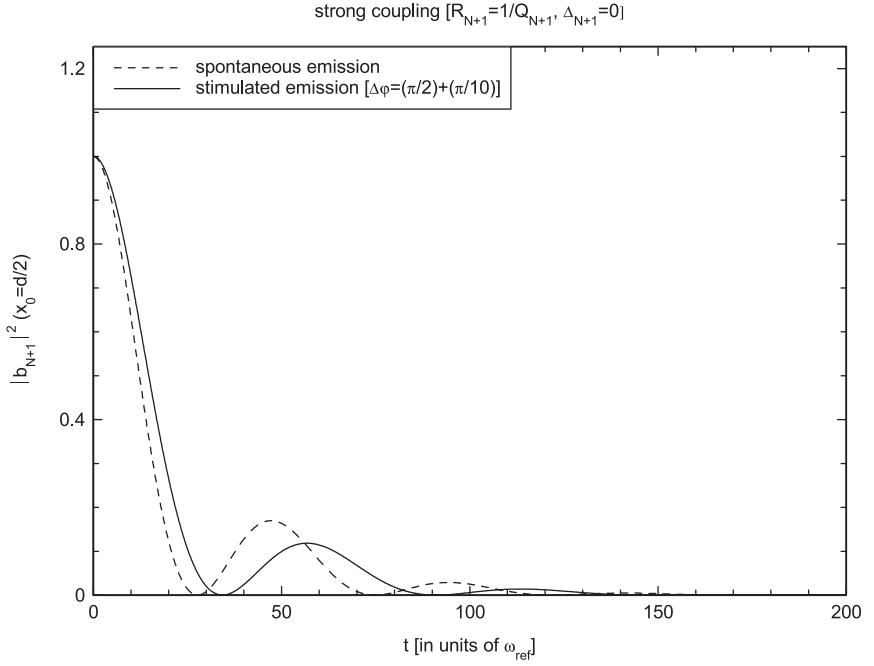

(a)

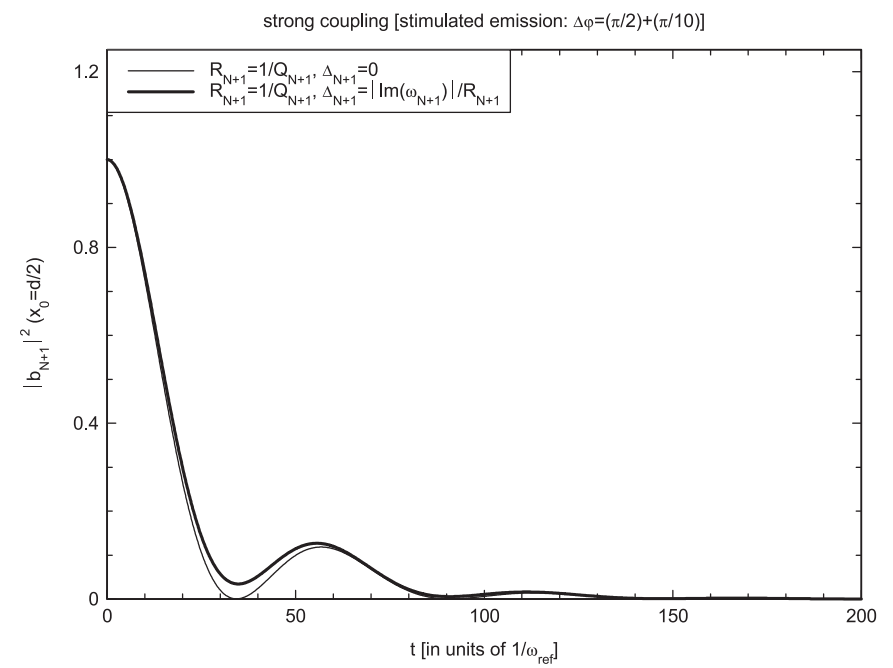

(b)

Fig. 4. Referring to the atom embedded inside the 1D-PBG cavity of Figure 1, the emission probability of the atom is plotted as a function of the normalized time $\omega_{\text {ref }}$, being $\omega_{\text {ref }}$ the 1D-PBG reference frequency. Referring to the operative conditions of Figure 3: in tuning hypothesis, as from Figure 4a, the atomic emission probability for spontaneous processes [see Eq. (5.7)] is compared with the stimulated emission probability [see Eq. (5.8)] when the 1D-PBG is pumped by two laser beams with a suitable phase difference: $\Delta \varphi=(\pi / 2)+(\pi / 10)$; in the case of stimulated processes, as from Figure $4 \mathrm{~b}$, the atomic emission probability in perfect tuning is compared with the emission probability in detuning case.

(Ref. [20]). Moreover, as discussed above, the phase difference must not exceed $\Delta \varphi_{1}=2.747$ (in units of rads), otherwise the Rabi splitting tends to zero; by increasing the phase difference with respect to $\Delta \varphi \approx \pi / 2$, in the time domain, the decay time becomes more long still and, in the frequency domain, the global transmission (7.1) is characterized by an high gain but the acceleration of coupling (7.2) presents a narrow pass band aspect. So, if $\Delta \varphi \rightarrow \Delta \varphi_{1}$, the active cavity acts as a delay line which is active but not ideal. Let conclude that the $1 \mathrm{D}-\mathrm{PBG}$ cavity should be pumped by two laser beams exceeding in quadrature of a tilt angle as:

$$
\Delta \varphi=\frac{\pi}{2}+\frac{\pi}{10}
$$




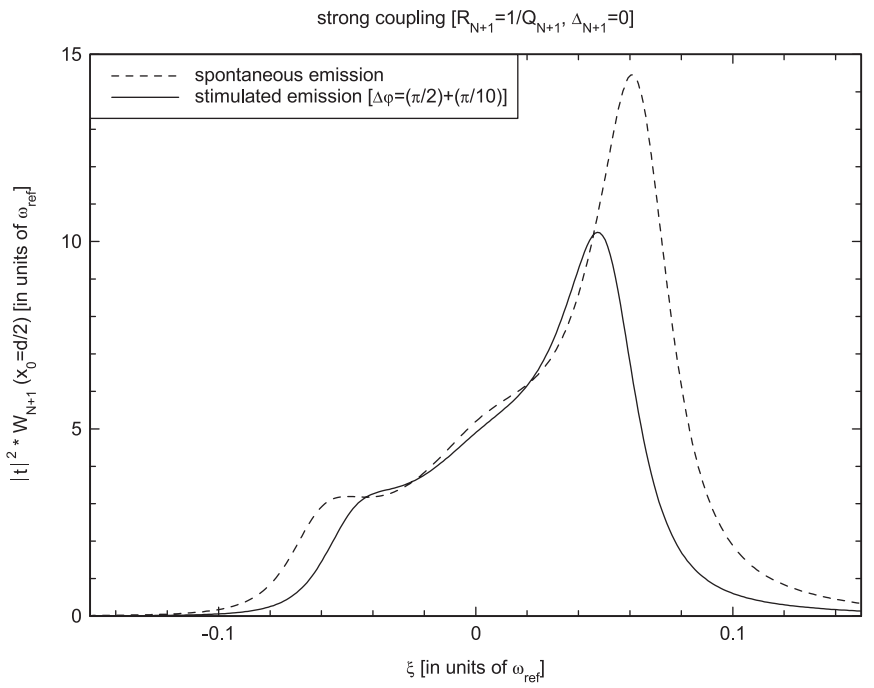

(a)

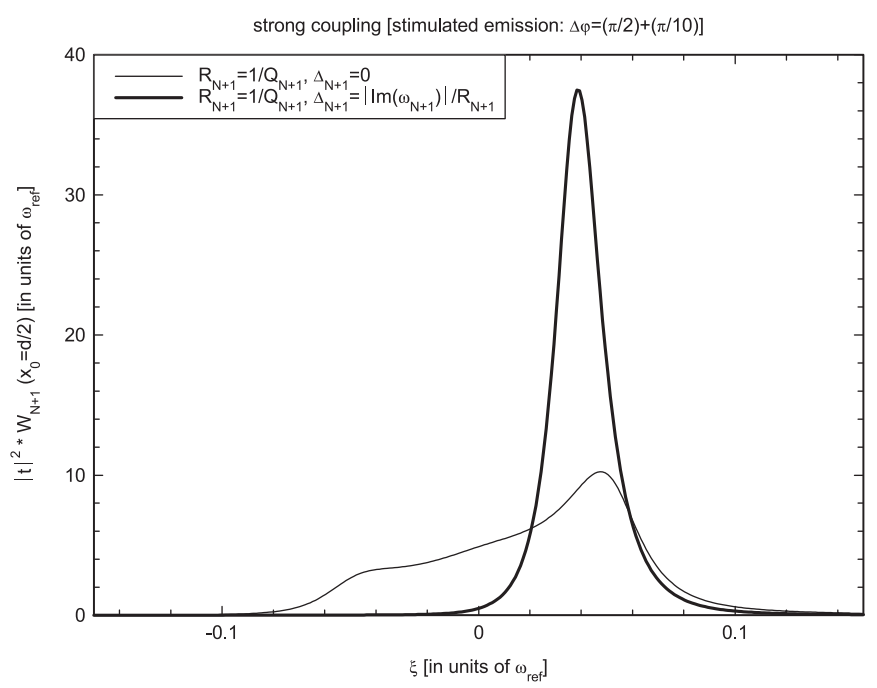

(c)

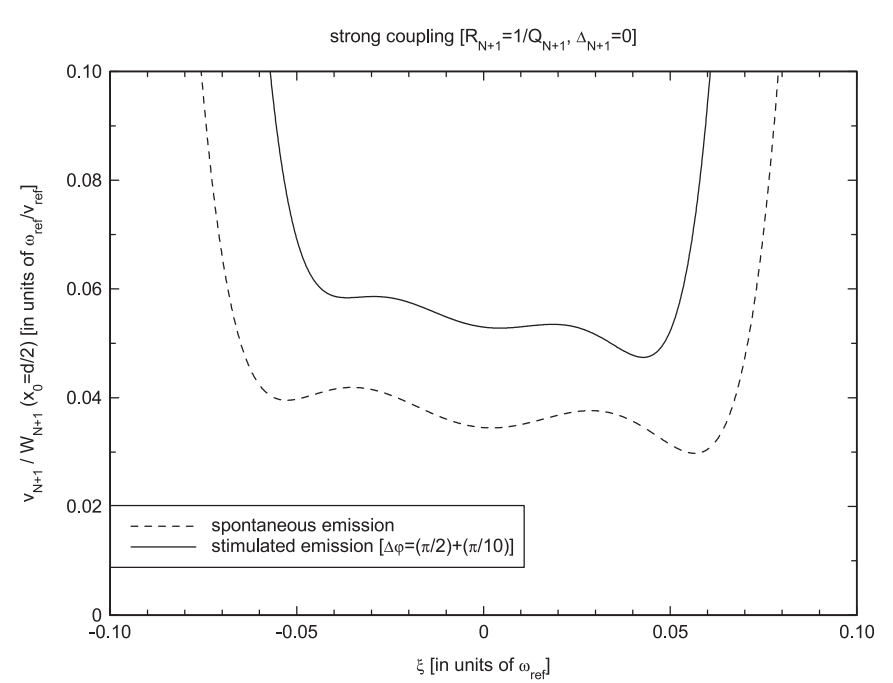

(b)

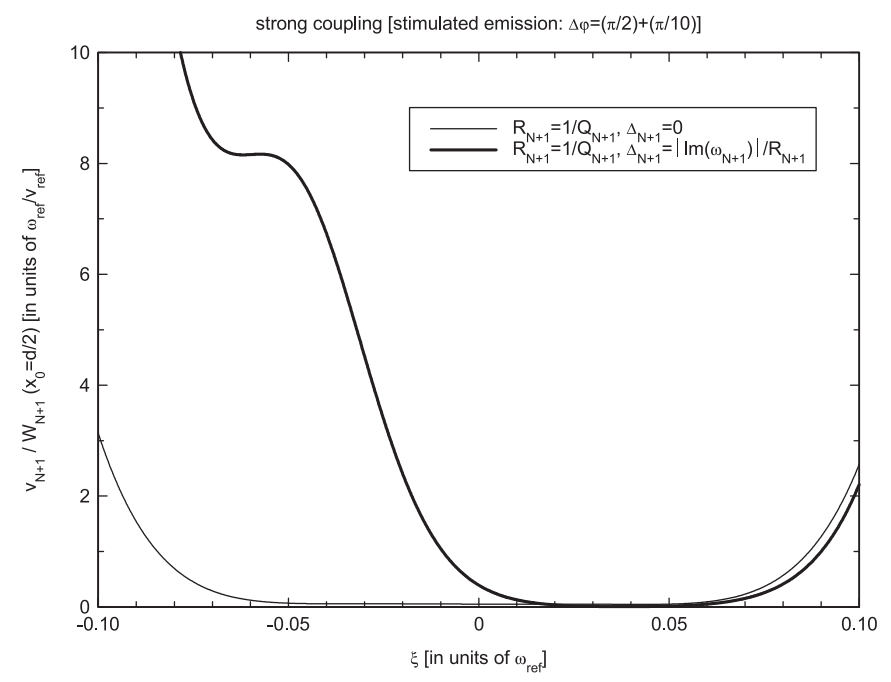

(d)

Fig. 5. In order to design a delay line using the active cavity composed of the 1D-PBG cavity plus the atom (Fig. 1), the delay line can be characterized by a global transmission [see Eq. (7.1)] and by a "coupling acceleration" [see Eq. (7.2)] of the e.m. field; the global transmission, in units of the 1D-PBG reference frequency $\omega_{r e f}$, and the coupling acceleration, in units of $\omega_{r e f} / v_{r e f}$, being $v_{r e f}$ the group velocity of the e.m. field in vacuum, are plotted as functions of the dimensionless shifted frequency $\xi=(\omega-\Omega) / \omega_{\text {ref }}$, being $\Omega$ the atomic resonance. Referring to the operative conditions of Figure 3: in perfect tuning, as from Figure 5a (Fig. 5b), the global transmission (the coupling acceleration) of the active delay line for spontaneous emission is compared with the global transmission (the coupling acceleration) for stimulated emission when the 1D-PBG is pumped by two laser beams with a suitable phase difference: $\Delta \varphi=(\pi / 2)+(\pi / 10)$; in the case of stimulated emission, as from Figure $5 \mathrm{c}$ (Fig. 5d), the global transmission (the coupling acceleration) of the active delay line in perfect tuning is compared with the global transmission (the coupling acceleration) in detuning case.

The two poles of the stimulated emission spectrum, shifted of the resonance $\Omega$, are $\xi_{1}=0.05205+i 0.01787$ and $\xi_{2}=-0.05205+i 0.01978$ (in units of $\omega_{\text {ref }}$ ) (see Figs. 2c and $2 \mathrm{~d}$ ); with respect to spontaneous emission, the two poles are closer by $\Delta \xi=0.02356$. They describe, in resonance and band width, the two peaks of the stimulated emission spectrum, whose maxima are $W_{1}=14.36$ and
$W_{2}=10.93$ (in units of $\omega_{r e f}$ ) (see Fig. 3a); with respect to spontaneous emission, the two maxima are lower by $\Delta W_{1}=7.51$ and $\Delta W_{2}=4.73$. Assuming the emission probability almost zero after the second oscillation, the decay time of stimulated emission is $\tau=113.5$ (in units of $1 / \omega_{\text {ref }}$ ) (see Fig. $4 \mathrm{a}$ ); with respect to spontaneous emission, this time is longer by $\Delta \tau=19.2$. So, the phase 
difference of the two laser beams allows to control the decay time of the atom and then the delay time of the active cavity [20]. Now, a delay line has been designed, but it is not quite ideal. An input pulse is retarded, amplified but a little distorted; as plotted in Figures $5 \mathrm{a}$ and $5 \mathrm{~b}$, with respect to spontaneous emission, there exists a narrower pass band, i.e. $\xi=\omega-\Omega \approx(-0.04,0.04)$, where the global transmission spectrum assumes similar values, i.e. $G_{C, N+1} \in\left(G_{\min }, G_{\max }\right)=(3.270,9.24)$ (in units of $\left.\omega_{\text {ref }}\right)$, and, above all, the acceleration of coupling is subject to a slight modulation, now around the value $v_{C, N+1}=0.05310$ (in units of $\omega_{\text {ref }} / v_{\text {ref }}$ ).

Let us finally consider stimulated emission in presence of some detuning: the atom inside the symmetric QW 1D-PBG cavity is still coupled to the $(N+1)$ th QNM, but does not oscillate any more at the $(N+1)$ th QNM frequency. The design of the active delay line can be improved varying, as last degree of freedom, the frequency detuning for the atom - $(N+1)$ th QNM coupling (7.5). In order to improve the active delay line, we propose to apply the maximum detuning. The atomic resonance $\Omega$ is dropped inside the photonic band gap next to the $(N+1)$ th QNM frequency $\operatorname{Re}\left(\omega_{N}+1\right)$; the atom is still coupled only to the $(N+1)$ th QNM if the atomic resonance is at the most

$$
\Omega=\operatorname{Re} \omega_{N+1}-\left|\operatorname{Im} \omega_{N+1}\right|,
$$

so that the detuning is maximum:

$$
\Delta_{N+1}=\frac{\operatorname{Re} \omega_{N+1}-\Omega}{R_{N+1}}=\frac{\left|\operatorname{Im} \omega_{N+1}\right|}{R_{N+1}} .
$$

The two poles of the stimulated emission spectrum in detuning case, shifted of the resonance $\Omega$, have the real parts $\operatorname{Re}\left(\xi_{1}\right)=0.03738$ and $\operatorname{Re}\left(\xi_{2}\right)=-0.07380$ and the imaginary parts $\operatorname{Im}\left(\xi_{1}\right)=0.01176$ and $\operatorname{Im}\left(\xi_{2}\right)=0.02588$ (both in units of $\omega_{\text {ref }}$ ) (see Figs. $2 \mathrm{c}$ and $2 \mathrm{~d}$ ); with respect to the perfect tuning, the real parts are lower by $\Delta \operatorname{Re}\left(\xi_{1}\right)=0.01467$ and $\Delta \operatorname{Re}\left(\xi_{2}\right)=0.02175$, while one imaginary part is lower by $\Delta \operatorname{Im}\left(\xi_{1}\right)=0.00611$ and the other is higher of $\Delta \operatorname{Im}\left(\xi_{2}\right)=0.0061$. They describe, in resonance and bandwidth, the two peaks of the stimulated emission spectrum in the detuning case, whose maxima are $W_{1}=38.83$ and $W_{2}=0.2974$ (in units of $\omega_{\text {ref }}$ ) (see Fig. $3 \mathrm{~b}$ ); with respect to the perfect tuning, the first peak is higher by $\Delta W_{1}=24.47$ and the second peak is lower by $\Delta W_{2}=10.63$. Assuming the atomic emission probability almost vanished after the second oscillation, the decay time of stimulated emission (linked the delay time of the active cavity) in the detuning case is $\tau=111.2$ (in units of $1 / \omega_{\text {ref }}$ ) (see Fig. $4 \mathrm{~b}$ ); with respect to the perfect tuning, the emission probability (and so the input pulse) is slightly warped and retarded by $\Delta \tau=2.3$.

At the end, an almost ideal delay line has been designed. An input pulse is retarded, amplified and almost not distorted; as plotted in Figures 5c and 5d, with respect to stimulated emission in detuning case, there exists a still narrower pass band, i.e. $\xi=\omega-\Omega \approx(0.02,0.06)$, where the global transmission spectrum assumes higher values, i.e. $G_{C, N+1} \in\left(G_{\min }, G_{\max }\right)=(6.005,36.77)$ (in units of $\left.1 / \omega_{\text {ref }}\right)$; above all, the acceleration of coupling is not subjected to any modulation, but is almost constant, i.e. $v_{C, N+1} \cong 0.007182$ (in units of $\omega_{\text {ref }} / v_{\text {ref }}$ ): as from Figure $5 \mathrm{~d}$, the modulation of the coupling acceleration is shifted over the not used frequency range $\xi \approx(-0.07,-0.04)$.

\section{Conclusions and discussion}

In this paper we have we have discussed in the strong coupling regime stimulated emission processes of an atom embedded inside a one dimensional (1D) Photonic Band Gap (PBG) cavity, pumped by two counter-propagating laser beams. Quantum electro-dynamics has been applied to model the atom-field interaction, by considering the atom as a two level system, the e.m. field in a superposition of its normal modes, the dipole approximation, the equations of motion of Wigner-Weisskopf and the rotating wave approximation. The Quasi Normal Mode (QNM) approach has been adopted for treating the open cavity situation under scrutiny, interpreting the local density of states (LDOS) as the local density of probability to excite one QNM in the cavity. In this way we get that this LDOS depends on the phase difference of the two laser beams as well as the main result that the strong coupling regime may be associated to high values of the LDOS. In addition we recover in the context of our approach the well known result [20], according to which the emission probability of the atom decays with oscillatory behaviour, so that the atomic emission spectrum is splitted into two peaks (Rabi splitting). The novelty of this paper is that the phase difference of the two laser beams might indeed provide a coherent control of both the oscillations for the atomic emission probability and the Rabi splitting for the emission spectrum. Finally, some criteria have been proposed to design the active cavity consisting of the 1D-PBG cavity plus the atom as an active delay line: suitable phase differences between the two laser beams allow to obtain a high transmission in a narrow pass band for a delayed pulse.

There are several possible approaches to the problem of electromagnetic field interaction with atoms when the e.m modes are affected by the environment (a cavity, or nearly walls). It is possible for example to solve the dynamics of the e.m. field inside and outside the cavity (or walls) at first, and then to treat the coupling of the atom with the normal modes (NMs) of the combined system. A second approach uses the discrete (dissipative) QNMs of the open cavity rather than the continuous (Hermitian) NMs. In the QNM treatment, the internal field cavity and the outside e.m. fields (out of the two sides of the cavity) are coupled by boundary conditions [22].

This paper proposes a third approach, a mixture of the first and second ones, in order to successfully blend their potentialities of analysis. In fact, the canonical quantum electrodynamics has been applied to express the e.m. field as a superposition of NMs, while the QNM approach has been adopted for an open cavity, interpreting the LDOS 
as the local density of probability to excite one QNM of the cavity. The DOS is related to the boundary conditions of the cavity. Since the e.m. field satisfies incoming and outgoing wave conditions on the surfaces of the cavity, the DOS depends on the photon reservoir due to the external pumping. If the cavity is excited by two counter propagating pumps, the DOS represents the probability distribution of exiting one QNM of the cavity.

If spontaneous emission processes occur, the two pumps are modelled as vacuum fluctuations, lying on the ground state of the e.m. field, and the DOS is only a peculiarity of the cavity geometry. In the case of stimulated emission, the two pumps are modelled as two laser beams, lying on a coherent state, so the DOS, besides depending on the geometry of the cavity, can be controlled by the phase difference of the two laser beams. These results put well into evidence how the DOS of an open cavity depends on the excitation condition of the cavity.

Work partially supported by Phoremost network of excellence.

\section{References}

1. E.M. Purcell, Phys. Rev. 69, 681 (1946); D. Kleppner, Phys. Rev. Lett. 47, 233 (1981)

2. K.H. Drexhage, Progress in Optics, edited by E. Wolf (North-Holland, New York, 1974), Vol. 12; P. Goy, J.M. Raimond, M. Gross, S. Haroche, Phys. Rev. Lett. 50, 1903 (1983); R.G. Hulet, E.S. Hilfer, D. Kleppner, Phys. Rev. Lett. 55, 2137 (1985); W. Jhe, A. Anderson, E.A. Hinds, D. Meschede, L. Moi, S. Haroche, Phys. Rev. Lett. 58, 666 (1987); D.J. Heinzen, J.J. Childs, J.E. Thomas, M.S. Feld, Phys. Rev. Lett. 58, 1320 (1987); F. De Martini, G. Innocenti, G.R. Jacobovitz, P. Mataloni, Phys. Rev. Lett. 59, 2955 (1987)

3. E.T. Jaynes, F.W. Cummings, Proc. IEEE 51, 89 (1963); G. Rempe, H. Walther, N. Klein, Phys. Rev. Lett. 58, 353 (1987)

4. S. John, Phys. Rev. Lett. 53, 2169 (1984)

5. E. Yablonovitch, Phys. Rev. Lett. 58, 2059 (1987); S. John, Phys. Rev. Lett. 58, 2486 (1987)

6. S. John, J. Wang, Phys. Rev. Lett. 64, 2418 (1990); Phys. Rev. B 43, 12772 (1991); S. John, Confined Electrons and Photons, edited by E. Burstein, C. Weisbuch (Plenum, New York, 1995); R.F. Nabiev, P. Yeh, J.J. Sanchez-Mondragon, Phys. Rev. A 47, 3380 (1993)
7. H.M. Lai, P.T. Leung, K. Young, Phys. Rev. A 37, 1597 (1988)

8. P.T. Leung, S.Y. Liu, K. Young, Phys. Rev. A 49, 3057 (1994); P.T. Leung, S.S. Tong, K. Young, J. Phys. A 30, 2139 (1997); P.T. Leung, S.S. Tong, K. Young, J. Phys. A 30, 2153 (1997); E.S.C. Ching, P.T. Leung, A. Maassen van der Brink, W.M. Suen, S.S. Tong, K. Young, Rev. Mod. Phys. 70, 1545 (1998)

9. A. Settimi, S. Severini, N. Mattiucci, C. Sibilia, M. Centini, G. D'Aguanno, M. Bertolotti, M. Scalora, M. Bloemer, C.M. Bowden, Phys. Rev. E 68, 026614 (2003); S. Severini, A. Settimi, C. Sibilia, M. Bertolotti, A. Napoli, A. Messina, Acta Phys. Hung. B, submitted, 20/1 (2005)

10. K.C. Ho, P.T. Leung, A. Maassen van den Brink, K. Young, Phys. Rev. E 58, 2965 (1998)

11. C. Cohen-Tannoudji, B. Diu, F. Laloe, Quantum Mechanics (John Wiley \& Sons, New York, 1977)

12. W.H. Louisell, Quantum Statistical Properties of Radiation (John Wiley \& Sons, New York, 1973)

13. S. Severini, A. Settimi, C. Sibilia, M. Bertolotti, A. Napoli, A. Messina, Phys. Rev. E 70, 056614 (2004)

14. S. Severini, A. Settimi, C. Sibilia, M. Bertolotti, A. Napoli, A. Messina, to be submitted to Laser Physics (2005)

15. A. Settimi, S. Severini, C. Sibilia, M. Bertolotti, M. Centini, A. Napoli, N. Messina, Phys. Rev. E 71, 066606 (2005)

16. A.A. Abrikosov, L.P. Gor'kov, I.E. Dzyaloshinski, Methods of Quantum Field Theory in Statistical Physics (Dover, Leung, A. Maassen van den Brink, K. Young, Frontiers in Quantum Physics, Proceedings of the International Conference, edited by S.C. Lim, R. Abd-Shukor, K.H. Kwek (Springer-Verlag, Singapore, 1998)

17. K.J. Blow, R. Loudon, S.J.D. Phoenix, T.J. Shepherd, Phys. Rev. A 42, 4102 (1990)

18. G.F. Carrier, M. Krook, C.E. Pearson, Functions of a complex variable - theory and technique (McGraw-Hill Book Company, New York, 1983)

19. S. Hughes, H. Kamada, Phys. Rev. B 70, 195313 (2004)

20. L. Allen, J.H. Eberly, Optical Resonance and Two-Level Atoms (Dover, New York, 1987); R. Bonifacio, L.A. Lugiato, Phys. Rev. A 11, 1507 (1975); K. Sakoda, J.W. Haus, Phys. Rev. A 68, 053809 (2003)

21. W.H. Louisell, L.R. Walker, Phys. Rev. 137, B204 (1965); I.R. Senitszky, Phys. Rev. 119, 670 (1960); 124, 642 (1961); 131, 2827 (1963); M. Lax, Phys. Rev. 145, 110 (1966)

22. H. Dekker, Phys. Lett. A 104, 72 (1984); H. Dekker, Phys. Lett. A 105, 395 (1984); H. Dekker, Phys. Lett. A 105, 401 (1984); H. Dekker, Phys. Rev. A 31, 1607 (1985) 